\title{
Visual Servoing for Intraoperative Positioning and Repositioning of Mobile C-arms
}

\author{
Nassir Navab ${ }^{1}$, Stefan Wiesner ${ }^{1}$, Selim Benhimane ${ }^{1}$, Ekkehard Euler ${ }^{2}$, \\ and Sandro Michael Heining ${ }^{2}$ \\ ${ }^{1}$ Chair for Computer Aided Medical Procedures (CAMP), TU Munich, Germany \\ ${ }^{2}$ Chirurgische Klinik und Poliklinik - Innenstadt, LMU Munich, Germany
}

\begin{abstract}
The problem of positioning mobile C-arms, e.g. for down the beam techniques, as well as repositioning during surgical procedures currently requires time, skill and additional radiation. This paper uses a Camera-Augmented Mobile C-arm (CAMC) to speed up the procedure, simplify its execution and reduce the necessary radiation. For positioning the $\mathrm{C}$-arm in down-the-beam position, the pre-operative diagnostic CT is used for defining the axis. Additional CT visible markers on patient's skin allow the CAMC's optical camera to compute the C-arm's pose and its required displacement for positioning. In the absence of electronically controlled mobile C-arms, the system provides step-by-step guidance to surgical staff until the final position is achieved. At this point, the surgeon can acquire an X-ray to ensure the correct positioning. In the case of intra-operative repositioning, no pre-operative $\mathrm{CT}$ is required. $\mathrm{X}$-ray/Optical markers allow the visual servoing algorithm to guide the surgical staff in C-arm repositioning using CAMC's optical camera. This work paves the path for many possible applications of visual servoing in $\mathrm{C}$-arm positioning and in surgical navigation. Experiments on phantom and a cadaver study demonstrate the advantages of the new methods.
\end{abstract}

\section{Introduction}

The mobile C-arm is an essential everyday tool in routine trauma and orthopaedic surgery. With increasing numbers of minimally invasive procedures the importance of fluoroscopic guidance is still growing, thus augmenting radiation doses $1 \begin{array}{lll}1 & 2 & 3\end{array}$. Considerable effort has been undertaken to improve the possibilities of C-arm imaging. Nowadays intraoperative 3D-imaging and C-arm based surgical navigation systems are commercially available. However in the surgical workflow, 3D-imaging is only possible at distinct points during the intervention e.g. to visualize the quality of fracture-reduction or to control the position of implants. During 3D image acquisition no manipulation like drilling or implant positioning is possible, i.e. the different steps of the surgical procedure are still carried out under 2D fluoroscopic imaging. So radiation exposure both to the patient and the surgical staff is often inevitable. In some surgical procedures even the direct exposure of the surgeon's hand cannot be avoided.

In the last decade the first medical augmented reality (AR) systems were introduced 56178910 . Most of the systems augment the view of the surgeon 
or an external camera with registered pre-operative data. Four Medical AR systems were also proposed that directly augment the intra-operatively acquired data 11,12 13 14. Camera-Augmented Mobile system is one of these systems, which attaches an optical camera to the X-ray source housing. Thanks to a double mirror system and an adopted one time calibration procedure performed during the construction of the system, the X-ray and optical images are aligned for all simultaneous acquisitions. If the patient does not move, the video images remain aligned with the X-ray image. This makes the system quite interesting for medical applications. We have built a new CAMC system based on the description provided in 1115. We have also demonstrated the efficiency of this video augmented X-ray fluoroscopy system for pedicale screw placement through a cadaver study [16. This work aims at the use of this newly built system for positioning and repositioning of the mobile C-arms during surgical procedures. The main contribution here is the use of visual servoing with no radiation for positioning a mobile $\mathrm{C}$-arm to obtain target images defined for the X-ray imaging. We have focused on the two following tasks that are often performed in various surgical applications:

Down-the-beam positioning. This task is often performed when there is a need for inserting a linear surgical tool to reach a deep seated anatomical target or to position a screw into an interlocking hole. It consists of aligning the entry point and a deep seated target, or the axis of perforation with the X-ray source such that the linear surgical tool or the axis of perforation is projected into a single point or the interlocking hole is presented with maximum apparent diameter on the X-ray image. Clinical examples for this "down-the-beam"-technique include, but are not limited to, the distal interlocking of intramedullary implants in the treatment of long bone fractures [17], the placement of transpedicular screws in spinal surgery [1816] and precise needle placement.

Intraoperative repositioning of mobile C-arm. During the course of an intervention, the mobile $\mathrm{C}$-arm has to be moved by OR-staff. These movements include bringing the $\mathrm{C}$-arm into the operation field and out again at certain points of the procedure, when fluoroscopic control is needed as well as the positioning for specific projections in regard to the anatomy, e.g. joint lines. For the control of the correct positioning, supporting systems such as goniometers or laser-visors are used. However additional fluoroscopic images cannot be avoided.

In this paper, we propose two visual servoing algorithms providing elegant solutions for performing the above two tasks with no additional radiation for surgeon as well as for the patient. Experimental results prove the efficiency of the algorithms.

\section{Overview of Methods}

In general, we attack the problem of finding a predefined position for the mobile $\mathrm{C}$-arm with respect to the patient. Markers are attached to the patient, which will be used as features for the visual servoing tasks. 
The repositioning task is done by (optical) image-based visual servoing. A target image from the current $\mathrm{C}$-arm position is taken. When the $\mathrm{C}$-arm is repositioned, the current positions of the fiducials are compared to the target positions and joint increments of the C-arm are computed (see figure 1).

For Down-the-Beam positioning of the C-arm, we use a pre-operative CT to define the Down-the-Beam axis. Intra-operatively we estimate the current pose of the $\mathrm{C}$-arm with regards to the CT data (see figure 21). Position-based visual servoing is used to move the C-arm towards the desired position. When we are close to the optimal position, we switch to image-based visual servoing with the target image generated from the CT data .

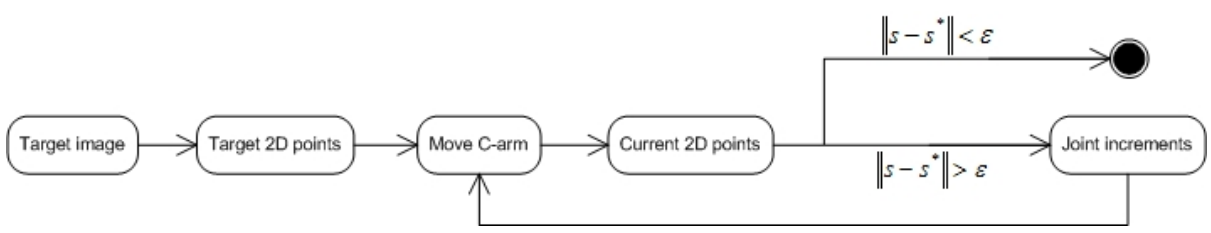

Fig. 1. intra-operative repositioning

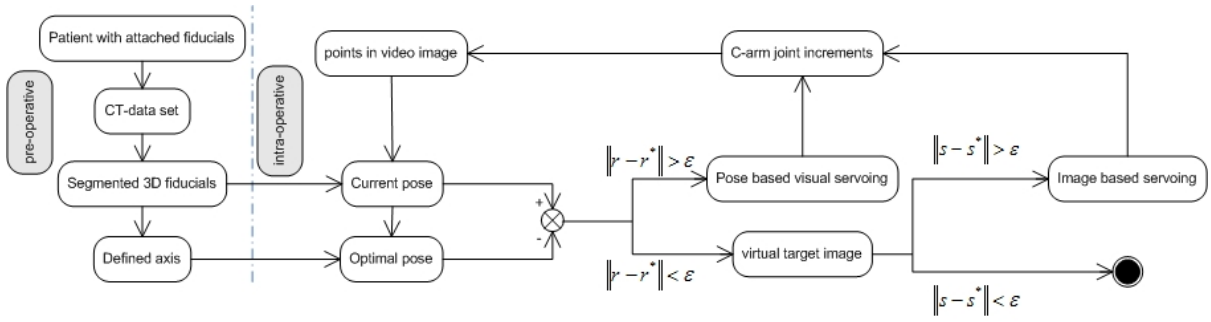

Fig. 2. Down-the-Beam C-arm positioning

\section{$3 \quad$ Visual Servoing}

Visual servoing aims to control the C-arm using the visual information taken from the on-board camera observing the scene at the current position $\mathcal{F}$ such that it reaches the desired (or the reference) position $\mathcal{F}^{*}$. If we denote by $\mathbf{q}$ the vector containing the joints current position, the objective of visual servoing is then to compute the direction and the amplitude of the increments of the joints, that correspond to $\dot{\mathbf{q}}$, in order to accomplish the positioning task. Consequently, we need to model the forward kinematics and the Jacobian of the C-arm.

\subsection{C-arm Motion Model}

In Figure 3, we sketched the forward kinematics of the C-arm (a similar kinematic analysis has also been done in [19]). The C-arm has 5 degrees of freedom: 


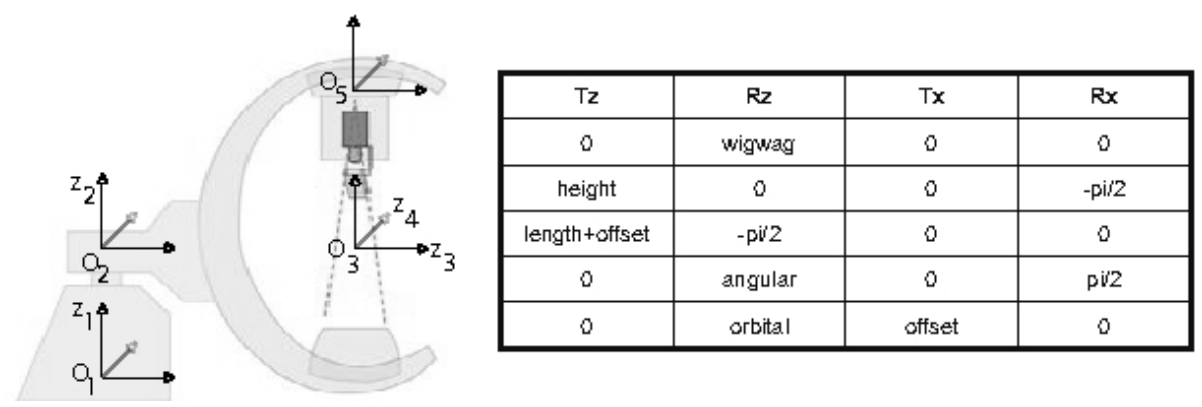

Fig. 3. C-arm coordinate frames

the height, the wigwag-movement, the length, the angular and the orbital movement. We assigned a coordinate frame to each joint according to the DenavitHartenberg rules [20. Let ${ }^{i} \mathbf{A}_{i+1}$ be the $(4 \times 4)$ transformation matrix from the coordinate system of the joint $i+1$ to the coordinate system of the joint $i$. Multiplying the different transformation matrices makes it possible to obtain the camera pose wrt. the world reference frame: ${ }^{0} \mathbf{A}_{6}={ }^{0} \mathbf{A}_{1}{ }^{1} \mathbf{A}_{2}{ }^{2} \mathbf{A}_{3}{ }^{3} \mathbf{A}_{4}{ }^{4} \mathbf{A}_{5}{ }^{5} \mathbf{A}_{6}$ The transformation matrix ${ }^{5} \mathbf{A}_{6}$ takes into account the rotation between the coordinate system of the last joint and the coordinate system of the camera.

To relate the motion of the camera to the motion of the $\mathrm{C}$-arm joints, we have to compute the manipulator Jacobian $\mathbf{J}_{\text {carm }}$ of the C-arm. It converts the velocities of the single joints $\dot{\mathbf{q}}$ to the Cartesian velocity of the camera $\mathbf{v}_{c}$.

The C-arm consists of 5 joints so the manipulator Jacobian is a $6 \times 5$ matrix. Its entries can be derived from the forward kinematics. The rotation axis $\mathbf{z}_{i}$ of each joint is found in the 3 rd column of the matrix ${ }^{0} \mathbf{A}_{i}={ }^{0} \mathbf{A}_{1} \ldots{ }^{i-1} \mathbf{A}_{i}$, where the origin $\mathbf{o}_{i}$ is found in the 4 th column. The axis and origins are visualized in Figure 3. Equation 1 lists first the column of the manipulator Jacobian for a prismatic joint (like length and height of the C-arm) and second for a revolute joint (like wigwag, angular and orbital movement).

$$
\mathbf{j}_{i}=\left(\begin{array}{c}
\mathbf{z}_{\boldsymbol{i}} \\
\mathbf{0}
\end{array}\right) ; \mathbf{j}_{i}=\left(\begin{array}{c}
\mathbf{z}_{\boldsymbol{i}} \times\left(\mathbf{o}_{5}-\mathbf{o}_{i}\right) \\
\mathbf{z}_{\boldsymbol{i}}
\end{array}\right)
$$

\subsection{Camera Model}

At the desired position $\mathcal{F}^{*}$, a $3 \mathrm{D}$ point $\mathcal{X}$ is projected on a virtual plane perpendicular to the optical axis of the camera into a $2 \mathrm{D}$ point $\mathbf{m}^{*}$ :

$$
\mathbf{m}^{*}=\left(x^{*}, y^{*}, 1\right) \propto\left[\mathbf{I}_{3 \times 3} \mathbf{0}_{3 \times 1}\right] \mathcal{X}
$$

The same $3 \mathrm{D}$ point $\mathcal{X}$ is projected into a $2 \mathrm{D}$ point $\mathbf{m}$ in the current camera position $\mathcal{F}: \mathbf{m}=(x, y, 1) \propto[\mathbf{R} \mathbf{t}] \mathcal{X}$ where $\mathbf{R}$ is the rotation matrix and $\mathbf{t}$ is the translation vector between the two coordinate systems $\mathcal{F}$ and $\mathcal{F}^{*}$. The information given by a pinhole camera, which performs a perspective projection of $3 \mathrm{D}$ points, is an image point $\mathbf{p}=(u, v, 1)$ verifying $\mathbf{p}=\mathbf{K} \mathbf{m}$ where $\mathbf{K}$ is the camera internal parameter matrix. 


\subsection{Pure Visual Servoing Using 2D Target Image}

The pure visual servoing using $2 \mathrm{D}$ target image can be done by making a vector s, containing visual information extracted from the current acquired image (at the current position $\mathcal{F}$ ), converging to a vector $\mathbf{s}^{*}$ containing visual information extracted from the reference image (at the reference position $\mathcal{F}^{*}$ ). In our case, the visual information are the image coordinates of the $n$ markers:

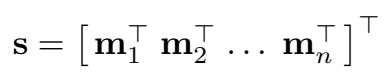

An interaction matrix $\mathbf{L}$ is then defined in order to establish the relationship between the Cartesian velocity of the camera $\mathbf{v}_{c}$ and the derivative of the vector $\mathbf{s}$ wrt. time. This relationship can be written as: $\dot{\mathbf{s}}=\mathbf{L v}_{c}$. The $(3 n \times 6)$ interaction matrix $\mathbf{L}$ can be expressed with the following formula: $\mathbf{L}=\left[\begin{array}{llll}\mathbf{L}_{1}^{\top} & \mathbf{L}_{2}^{\top} & \ldots & \mathbf{L}_{n}^{\top}\end{array}\right]^{\top}$ where:

$$
\mathbf{L}_{i}=\left[\begin{array}{cccccc}
\frac{1}{Z_{i}} & 0 & -\frac{x_{i}}{Z_{i}} & x_{i} y_{i} & -\left(1+x_{i}^{2}\right) & y_{i} \\
0 & \frac{1}{Z_{i}} & -\frac{y_{i}}{Z_{i}} & \left(1+y_{i}^{2}\right) & -x_{i} y_{i} & -x_{i} \\
0 & 0 & 0 & 0 & 0 & 0
\end{array}\right]
$$

The vector $\mathbf{s}$ is servoed using the task function approach [21|22] by minimizing iteratively the following vector $\mathbf{e}=\widehat{\mathbf{L}}^{+}\left(\mathbf{s}-\mathbf{s}^{*}\right)$, where $\widehat{\mathbf{L}}^{+}$is an approximation of the pseudo-inverse of the true interaction matrix $\mathbf{L}^{+}=\left(\mathbf{L}^{\top} \mathbf{L}\right)^{-1} \mathbf{L}^{\top}$. If we differentiate this equation, we obtain:

$$
\dot{\mathbf{e}}=\frac{d \widehat{\mathbf{L}}^{+}}{d t}\left(\mathbf{s}-\mathbf{s}^{*}\right)+\widehat{\mathbf{L}}^{+} \dot{\mathbf{s}}=\left(\mathbf{O}\left(\mathbf{s}-\mathbf{s}^{*}\right)+\widehat{\mathbf{L}}^{+} \mathbf{L}\right) \mathbf{v}_{c}
$$

where $\mathbf{O}\left(\mathbf{s}-\mathbf{s}^{*}\right)$ is a $(6 \times 6)$ matrix that can be neglected for $\mathbf{s} \approx \mathbf{s}^{*}$. Let's consider the control law: $\mathbf{v}_{c}=-\lambda \mathbf{e}$ where $\lambda$ is a positive scalar. Plugging this equation into equation (44), we obtain the following closed-loop equation: $\dot{\mathbf{e}}=-\lambda\left(\mathbf{O}\left(\mathbf{s}-\mathbf{s}^{*}\right)+\widehat{\mathbf{L}}^{+} \mathbf{L}\right) \mathbf{e}$. It is well known from the control theory that this non-linear system is locally asymptotically stable in a neighborhood of $\mathbf{s}=$ $\mathbf{s}^{*}$, if and only if, the matrix $\widehat{\mathbf{L}}^{+} \mathbf{L}$ has eigenvalues with a positive real part $\operatorname{real}\left(\operatorname{eig}\left(\widehat{\mathbf{L}}^{+} \mathbf{L}\right)\right)>0$. Obviously, if the pseudo-inverse of the interaction matrix is well approximated, we have: $\widehat{\mathbf{L}}^{+} \mathbf{L} \approx \mathbf{I}$ and the control law is locally asymptotically stable. Then, using the camera Cartesian velocity $\mathbf{v}_{c}$, it is possible to compute the joints increments $\dot{\mathbf{q}}$ given the pseudo-inverse of the C-arm Jacobian:

$$
\dot{\mathbf{q}}=\mathbf{J}_{\text {carm }}^{+} \mathbf{v}_{c}
$$

\subsection{Position-Based Visual servoing}

Figure 4 a shows the algorithm to find the optimal C-arm pose. First the initial C-arm pose ${ }^{w} \mathbf{T}_{c}$ is set manually by inputting a rough estimate of the initial joint values, since no sensors are attached to the $\mathrm{C}$-arm, which report the current pose. Then we estimate the current pose ${ }^{c} \mathbf{T}_{c t}$ of the camera wrt. to the frame of the CT-data set. The data is now transformed into the world frame $\mathcal{X}_{w}={ }^{w} \mathbf{T}_{c}$ 

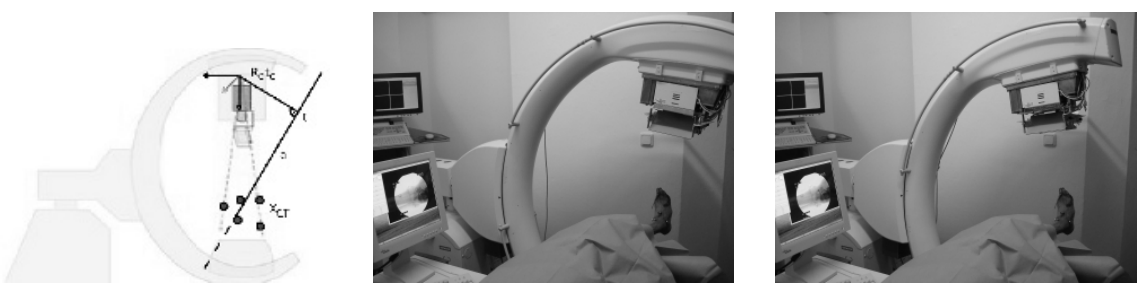

Fig. 4. (a) Projection of current camera center onto defined axis (b) reference position and start position of the $\mathrm{C}$-arm

${ }^{c} \mathbf{T}_{c t} \mathcal{X}_{c t}$ To get a first approximation of the optimal camera position, the camera center $\mathbf{t}$ is projected onto the defined axis $\mathbf{a}$. This gives the new camera center $\mathbf{t}^{*}$. The translational velocity is then set such that it is proportional to the difference between the current and the desired coordinate system center. While the rotational velocity $\boldsymbol{\omega}$ is computed thanks to the cross-product between the current camera optical axis $\mathbf{z}$ and the axis $\mathbf{a}: \boldsymbol{\nu}=-\lambda_{\nu}\left(\mathbf{t}-\mathbf{t}^{*}\right)$ and $\boldsymbol{\omega}=-\lambda_{\omega}(\mathbf{a} \times$ z) We now assemble them into the velocity screw. The joint increments are computed by equation 5. Now the C-arm is moved according to the computed parameters and the optimal position is estimated again. We stop the iterations when we are near the optimal position and generate a target image which is used for the final adjustment based on image based visual servoing.

\subsection{Generation of the Target Image}

In order to generate a virtual target image, we set up a virtual camera at the position $\mathbf{T}^{*}$ : with a the direction of the segmented axis, $\mathbf{u}$ and $\mathbf{v}$ verify $\mathbf{u} \times \mathbf{v}=\mathbf{a}$ and $\mathbf{p}$ is a point on the axis. The remaining parameters, a rotation $\theta$ around and a translation $t$ along the segmented axis are encapsulated in the transformation matrix $\mathbf{T}(\theta, t)$

$$
\mathbf{T}^{*}=\left[\begin{array}{llll}
\mathbf{u} & \mathbf{v} & \mathbf{a} & \mathbf{p} \\
0 & 0 & 0 & 1
\end{array}\right] \text { and } \mathbf{T}(\theta, t)=\left[\begin{array}{cccc}
\cos (\theta) & -\sin (\theta) & 0 & 0 \\
\sin (\theta) & \cos (\theta) & 0 & 0 \\
0 & 0 & 1 & t \\
0 & 0 & 0 & 1
\end{array}\right]
$$

The parameters are chosen such that they minimize the cost function $\min _{\theta, t}\left\|\mathbf{s}-\mathbf{s}^{*}(\theta, t)\right\|$ where $\mathbf{s}$ contains the current image points (given by the equation (3) ) and $\mathbf{s}^{*}(\theta, t)$ contains the projection of the computed $3 \mathrm{D}$ points

$$
\mathbf{s}^{*}(\theta, t)=\left[\mathbf{m}_{1}^{* \top}(\theta, t) \mathbf{m}_{2}^{* \top}(\theta, t) \ldots \mathbf{m}_{n}^{* \top}(\theta, t)\right]^{\top}
$$

with $\forall i, \mathbf{m}_{i}^{*}(\theta, t)$ is the projection of the $\mathrm{i}$-th 3D point given by the CT-data set and transformed by $\mathbf{T}^{*} \mathbf{T}(\theta, t)$. 


\section{Experiments}

\subsection{Down-the-Beam Positioning}

A spine phantom was placed under the C-arm in neutral position. The pose is estimated and the video image is augmented with the preoperative segmented data (fiducials and axis). Following the instruction of the servoing algorithm we iteratively changed the joint parameters. Figure 5(1-4) shows the acquired images during position based guidance. We performed first an orbital movement, a length change, an angular movement and finally again a length change. Being close to the optimal C-arm position we generated the target image and superimposed it onto the video image. Fine adjustment brings the C-arm to the final position, where an X-ray is taken and blended into the view. Now the placement of the screw is performed down the beam (see figure 5).
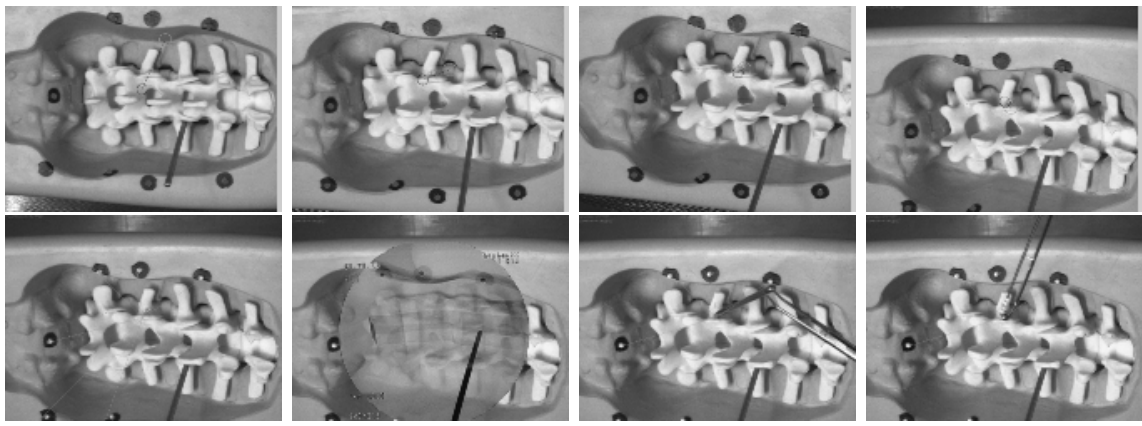

Fig. 5. position based servoing; 1: start image; 2: orbital and length change; 3: angulation change; 4:length change 5: target image superimposition; 6 : length changed and X-ray superimposition; 7,8: placement of pedicle screw

\subsection{C-arm Repositioning: Cadaver Study with Ankle Joint}

The standard radiographic examination of the ankle for fracture detection consists of the ap, mortise and lateral view. Intraoperatively the precise mortise view is mandatory to proof the correct reposition of the ankle. This view can be achieved at approximately $20^{\circ}$ internal rotation. The $\mathrm{C}$-arm was positioned by the surgeon according to the intervention (ap,mortise). After that we moved the $\mathrm{C}$-arm position about $-20^{\circ}$ in the orbital direction and $-5^{\circ}$ in the angular one (figure $4 \mathrm{~b}$ ). To reposition the $\mathrm{C}$-arm we followed the instruction of the visual servoing algorithm. Fine adjustment was done by comparing the current fiducial position with the target ones. C-arm reposition was performed according to the computed instructions: start with orbital movement, followed by a wigwag motion. The next iteration computed an angular movement and a wigwag movement. The reference X-ray and the resulting X-ray acquired after repositioning are fused with a checkerboard blending (Figure 7) to verify the result. 

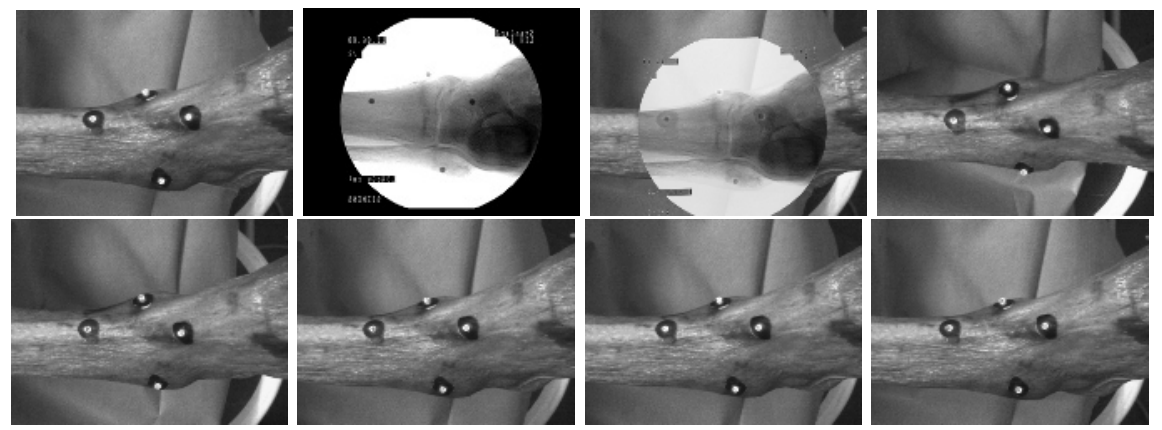

Fig. 6. Ankle joint repositioning; 1: reference video; 2: reference X-ray; 3: blended reference images; 4-8: images acquired during movement from start to reference position of C-arm

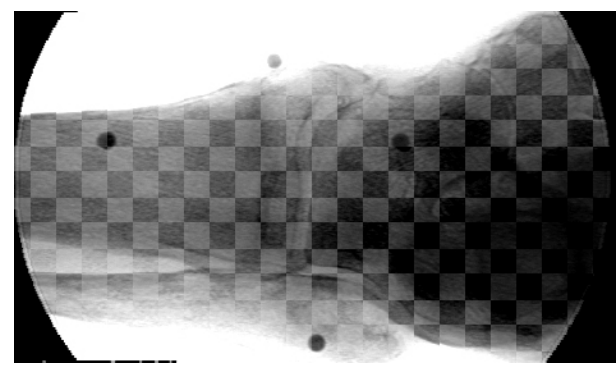

Fig. 7. Checkerboard fusion of reference X-ray and repositioning X-ray

\section{Discussion}

The presented positioning algorithm proved to be robust and effective in a clinical setup (pedicle screw placement and ORIF of ankle fracture). Computation is fast (real-time) and thus easily acceptable for clinical applications. After guidance with help of the algorithm interactive visual servoing allows high precision at small incremental steps. The number of positioning steps is comparable to clinical routine but without any radiation exposure. However X-ray images can be obtained at any time to check the reached position or to check patient movement. On one hand, the system fixes user's errors and takes patient's movements into account by systematically recomputing the appropriate trajectory at each step. On the other hand since the surgeon is also in the loop, rare potential risks, such as collision with the OR table, are prevented. The solution is intuitive and can be easy integrated into the clinical workflow. 


\section{Conclusion}

We proposed a visual servoing approach for positioning and repositioning of the mobile X-ray C-arm with attached and co-registered optical camera. The Carm geometry and all its degrees of freedom are modelled and two solutions for position based and image based visual servoing are proposed. We have tested the system in collaboration with a trauma surgeon in two experiments, the first one on a phantom and the second one on a cadaver. Interestingly, the surgeon prefers the interactive solution more than a possible full automatic one, since the process is quite fast and he is in full control. The system allows frequent tasks such as C-arm repositioning and down-the-beam positioning to be performed with minimum radiation for patient and no radiation for physician.

\section{References}

1. Boszczyk, B.M., et al.: Fluoroscopic radiation exposure of the kyphoplasty patient. European Spine Journal (2006) 347 - 355

2. Synowitz, M., Kiwit, J.: Surgeon's radiation exposure during percutaneous vertebroplasty. J Neurosurg Spine. 4(2) (2006) 106-109

3. Rampersaud, Y.R., et al.: Radiation exposure to the spine surgeon during fluoroscopically assisted pedicle screw insertion. Spine. 25(20) (2000) 2637-2645

4. Theocharopoulos, N., et al.: Occupational exposure from common fluoroscopic projections used in orthopaedic surgery. The Journal of Bone and Joint Surgery (American) 85 (2003) 1698-1703

5. State, A., Chen, D.T., Tector, C., Brandt, A., Chen, H., Ohbuchi, R., Bajura, M., Fuchs, H.: Case study: observing a volume rendered fetus within a pregnant patient. In: Proceedings of the conference on Visualization '94, IEEE Computer Society Press (1994) 364-368

6. Grimson, W., Ettinger, G., Kapur, T., M.E. Leventon, W.M. Wells, R.K.: Utilizing segmented mri data in image guided surgery. International Journal of Pattern Recognition and Artificial Intelligence (1996)

7. P.J.Edwards, Hill, D.D., Hawkes, D.D., Colchester, D.A.: Neurosurgical guidance using the stereo microscope. In: Proceedings of Computer Vision, Virtual Reality and Robotics in Medicine, First International Conference, CVRMed'95. (1995)

8. Blackwell, M., Nikou, C., Gioia, A.M.D., Kanade, T.: An image overlay system for medical data visualization. (1998)

9. Sauer, F., Schoepf, U.J., Khamene, A., Vogt, S., Das, M., Silverman, S.G.: Augmented reality system for ct-guided interventions: System description and initial phantom trials. In: Medical Imaging: Visualization, Image-Guided Procedures, and Display. (2003)

10. Birkfellner, W., Figl, M., Huber, K., Watzinger, F., Wanschitz, F., Hummel, J., Hanel, R., Greimel, W., Homolka, P., Ewers, R., Bergmann, H.: A head-mounted operating binocular for augmented reality visualization in medicinedesign and initial evaluation. IEEE Transaction on Medical Imaging 21(8) (2002) 991-997

11. Navab, N., Mitschke, M., Bani-Hashemi, A.: Merging visible and invisible: Two camera-augmented mobile C-arm (CAMC) applications. In: Proc. IEEE International Workshop on Augmented Reality, San Francisco, CA, USA (1999) 134-141

12. Stetten, G.D., et al.: C-mode real time tomographic reflection for a matrix array ultrasound sonic flashlight. (2003) 
13. Masamune, K., et al.: An image overlay system with enhanced reality for percutaneous therapy performed inside ct scanner. (2002)

14. Leven, J., et al.: DaVinci Canvas: A Telerobotic Surgical System with Integrated, Robot-Assisted, Laparoscopic Ultrasound Capability. In: MICCAI. (2005) 811-818

15. Mitschke, M., Bani-Hashemi, A., Navab, N.: Interventions under video-augmented X-ray guidance: Application to needle placement, Pittsburgh, PA, USA (2000) $858-868$

16. Heining, S., Euler, E., Wiesner, S., Navab, N.: Pedicle screw placement under video-augmented fluoroscopic control: First clinical application - a cadaver study. In: CARS 2006 - Computer Assisted Radiology and Surgery. (2006)

17. Whatling, G.M., Nokes, L.D.: Literature review of current techniques for the insertion of distal screws into intramedullary locking nails. Injury. (2005)

18. Hart, R.A., et al.: Pedicle screw placement in the thoracic spine: a comparison of image-guided and manual techniques in cadavers. Spine. 30(12) (2005) 326-331

19. Binder, N., Matthus, L., Burgkart, R., Schweikard, A.: A robotic c-arm fluoroscope. Int. Journal on Medical Robotics and Computer Assisted Surgery 1(3) (2005) 108-116

20. Denavit, J., Hartenberg, R.S.: A kinematic notation for lower-pair mechanisms based on matrices. Jour. of Applied Mechanics 22 (1955) 215-221

21. Samson, C., Le Borgne, M., Espiau, B.: Robot Control: the Task Function Approach. Oxford Engineering Science Series. Clarendon Press, Oxford, UK (1991)

22. Espiau, B., Chaumette, F., Rives, P.: A new approach to visual servoing in robotics. IEEE Trans. on Robotics and Automation 8(3) (1992) 313-326 